\title{
Quelle approche pour évaluer les résultats d'un projet d'enseignement à distance?
}

\author{
Jean-Marie Dujardin — Séverine Maron \\ HEC - Ecole de Gestion de l'Université de Liège \\ Rue Louvrex 14 \\ $B-4000$ LIEGE \\ Belgique \\ \{Jean-Marie.Dujardin; Severine.Maron\}@ulg.ac.be
}

RÉSUMÉ. Le Projet Campus Virtuel en Gestion a permis de développer depuis 2002 des cours en ligne dans le domaine du management. Les partenaires du projet se sont interrogés sur les effets tant économiques que pédagogiques de celui-ci. Les questions posées à travers cette étude de cas, sont les suivantes : - comment adopter une approche fondée sur l'évaluation tant économique que pédagogique d'un projet e-learning? - comment mener une telle évaluation? - quels sont les intérêts et les limites de la démarche? La méthodologie construite et présentée ici permet d'évaluer les aspects économiques et les aspects pédagogiques $d u$ projet. Les difficultés d'application, les intérêts, les limites de la méthodologie d'évaluation sont mis en évidence.

ABSTRACT. Since 2002 the so called "Campus Virtuel en Gestion" has developed on line courses in the field of management. The partners of the project wanted to know more about its results, from both a pedagogical and an economic point of view. The questions we address here are the following: - how to assess this project on both its economic and pedagogical effects? - how to carry out such an assessment? - what are the advantages and the limits of this approach? The difficulties of application, the interests, the limits of the methodology will be explained.

MOTS-CLÉS : e-learning, efficience, efficacité, évaluation économique, évaluation pédagogique KEYWORDS: e-learning, efficiency, effectiveness, economic evaluation, pedagogical evaluation. 
Le projet Campus Virtuel en Gestion $(C V G)$, collaboration entre trois écoles de gestion importantes en Communauté française de Belgique, Solvay Business School, IAG-Louvain School of Management, et HEC-Ecole de gestion de l'Université de Liège, a permis de développer depuis 2002 des cours en ligne de management. Ce projet est subventionné par le Fonds Social Européen ainsi que par la Communauté française de Belgique.

La préoccupation principale des partenaires a été, après cinq années de développement, d'une part, d'analyser les résultats globaux du projet, tant qualitatifs que quantitatifs, et, d'autre part, de mettre en relation ces résultats avec l'ensemble des ressources investies dans le projet. C'est avec cette optique que nous avons réalisé une analyse de la littérature et des projets européens concernant l'évaluation des projets e-learning et les approches qualité dans ce domaine, afin de construire une méthodologie d'évaluation du projet Campus Virtuel en Gestion.

$\checkmark$ L'approche de Hulsman (2003) analyse de manière systématique l'ensemble des coûts qui sont liés à un projet e-learning. Il distingue les coûts opérationnels et les coûts d'investissement. Les premiers comprennent les coûts directs de développement et d'animation des cours, ainsi que les coûts indirects relatifs au projet global. Les coûts d'investissement concernent l'expansion du programme et les coûts d'investissement en qualité

Supprimé : đI

Supprimé :

La démarche de Ljosa (2003) analyse l'ensemble des ressources (inputs) d'un projet e-learning et l'ensemble des résultats (outputs), dans une approche de type «boîte noire », c'est-à-dire sans tenir compte des processus et des activités mis en œuvre dans le projet. Les inputs sont: les contenus de cours, les compétences à atteindre, les technologies, les ressources en personnel, etc. Les outputs sont le nombre d'heures développées, le nombre d'heures dispensées, le nombre d'étudiants, les connaissances et compétences acquises, les crédits et diplômes obtenus, etc

- Rekkedal (2003) propose également une approche fondée sur les produits (résultats) d'un projet e-learning, mis en relation systématiquement avec les ressources nécessaires pour obtenir ces produits. Cette mise en relation systématique entre ressources et résultats est une forme d'analyse coût-efficacité du projet.

Holmberg (2003) invite quant à lui à mener une analyse coût-efficacité d'un projet en e-learning par comparaison avec un programme présentiel classique de manière à tester l'hypothèse selon laquelle un programme en e-learning, lorsqu'il s'adresse à une grande population d'étudiants, permet de générer des économies d'échelle par la répartition des coûts fixes sur un grand nombre d'apprenants

L'approche de Kirkpatrick (1994) considère quatre niveaux d'évaluation d'un programme de formation : la satisfaction des participants quant à son déroulement, 
les acquis en termes de compétences, le transfert au poste de travail, l'impact sur le fonctionnement et la performance de l'organisation analysée.

Le projet Leonardo Da Vinci SEVAQ (www.be-odl.org) vise à améliorer la qualité des prestations en e-learning en prenant en compte les besoins exprimés par les apprenants et en s'appuyant en particulier sur des feedback récoltés auprès de ceux-ci et dans leur environnement.

Le projet Qual e-learning (www.qual-elearning.net) mesure la qualité par l'analyse de la satisfaction des étudiants, de l'efficacité des programmes via des tests ou encore par le calcul du retour sur investissement des projets.

\section{La méthode d'évaluation}

Compte tenu des démarches d'évaluation évoquées et pour répondre aux attentes des parties prenantes du projet, c'est vers une analyse des inputs et des outputs à travers un tableau de bord d'un projet de e-learning que l'on s'est orientés. Celui-ci est présenté ci-dessous.

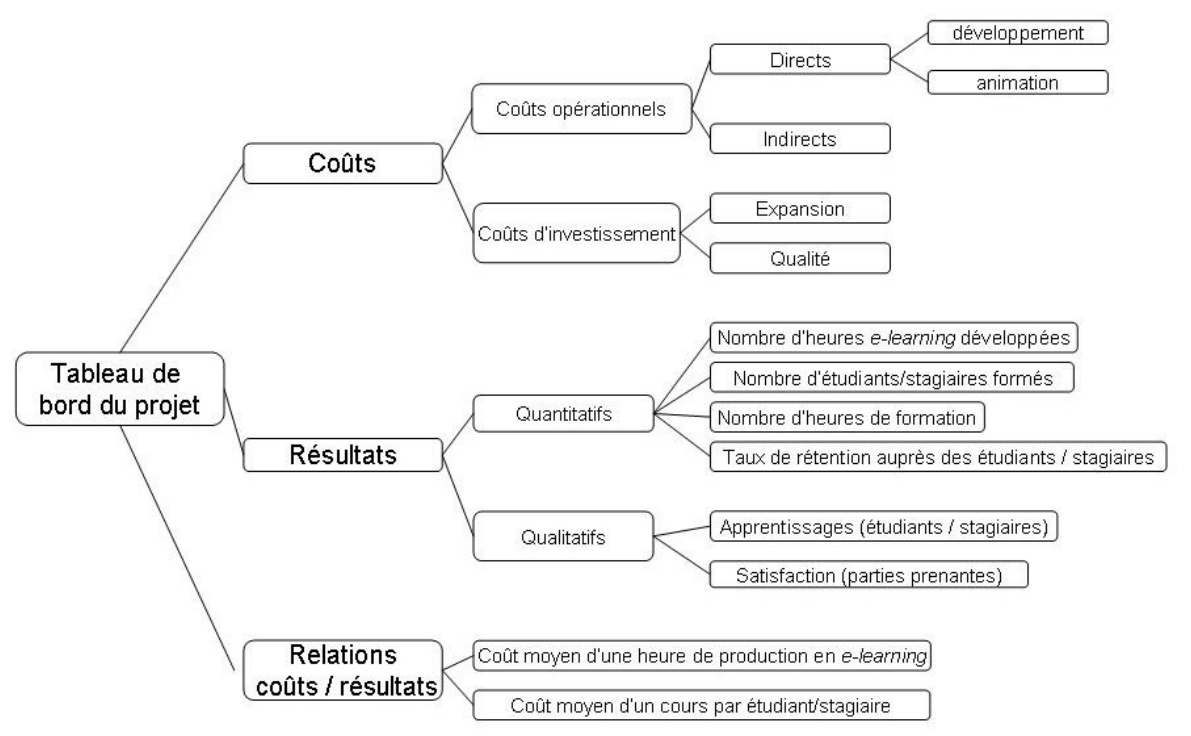

Figure 1. Tableau de bord du projet e-learning CVG

Tout d'abord, une analyse des ressources a été réalisée, en suivant l'approche de Hulsman(2003). On a distingué ainsi les coûts opérationnels des coûts 
d'investissement. Les premiers comprennent les coûts directs de développement et d'animation des cours. Les coûts indirects concernent les rémunérations de l'équipe projet, les frais de fonctionnement, les équipements locaux. Les coûts d'investissement concernent l'expansion du programme, comme le développement de nouveaux cours, et la qualité.

Ensuite, une analyse des résultats quantitatifs a été réalisée en termes de nombre d'heures développées, nombre d'heures de cours données, nombre de stagiaires ou d'étudiants, nombre d'heures/stagiaire réalisées, etc. Nous avons ainsi pu mettre en relation les inputs et les outputs en termes de coût moyen de production d'une heure en e-learning, coût moyen de prestation d'une heure en e-learning, coût moyen d'un cours ou d'une heure de cours par étudiant/stagiaire.

Dans l'évaluation des résultats, nous avons été également attentifs à l'analyse des apprentissages des étudiants/stagiaires, en termes de connaissances et compétences acquises, tant en fin de formation ( "évaluation à chaud ») qu'après un certain laps de temps, par exemple dix mois («évaluation à froid»). L'évaluation à froid a été menée tant auprès des étudiants/stagiaires qu'auprès de leur superviseur/responsable hiérarchique au travail. De même, la satisfaction des parties prenantes du projet a été analysée auprès des étudiants/stagiaires, des enseignants/développeurs, des employeurs. Nous avons apprécié tant la satisfaction « à chaud» que la satisfaction « à froid», par des méthodes de type questionnaire et/ou interviews

\section{Application de la méthode d'évaluation au projet CVG}

\section{Analyse des coûts}

Pour analyser les coûts liés au projet CVG sur la période 2002-2006, nous avons travaillé à deux niveaux : au niveau global du projet et au niveau de deux cours en particulier. Pour les coûts directs, nous avons relevé tous les coûts relatifs à un cours en particulier. Pour les coûts indirects, nous avons repris les coûts relatifs au projet dans sa globalité. Nous les avons répartis d'après l'importance des coûts directs des différents cours développés. Les coûts d'expansion du projet ont été calculés d'après une clé de répartition, qui consiste à considérer que le chef du projet consacrait $20 \%$ de son temps à la recherche de nouveaux cours, de nouveaux programmes, et de nouveaux financements. Les coûts d'investissement en qualité ont également été calculés d'après une clé de répartition, selon laquelle le chef du projet consacrait $10 \%$ de son temps à la maintenance et à l'évolution des cours, à la participation à des colloques et à la rédaction d'articles de recherche.

Pour cette analyse, il convient de décrire les données globales relatives aux activités du projet CVG. Entre 2002 et 2006, neuf cours ont été conçus, soit environ 350 heures d'e-learning. Tous ces cours ont été mis en œuvre à la fois en formation initiale (2 200 étudiants) et en formation continue au sein des trois écoles partenaires 
ou dans d'autres écoles (261 stagiaires), pour atteindre un nombre total de 66000 heures en formation initiale et de 12000 heures en formation continue. Entre 2002 et 2006, au niveau global, plus d'un million d'euros (1010500 €) ont été investis dans le projet CVG, toutes sources de financement confondues. Au niveau des cours analysés, le premier (un cours de gestion logistique démarré en 2002) a coûté au total près de $140000 €$ tandis que le second (un cours de «management des personnes », démarré en 2004) a coûté près de 70000 euros. Pour chacun des cours analysés les coûts opérationnels ont représenté environ $90 \%$ du total (répartis à parts égales entre coûts directs et indirects) et donc les coûts d'investissement ont représenté environ $10 \%$ (répartis également à parts quasi égales entre expansion et qualité). Des proportions quasi similaires peuvent être constatées au niveau des coûts de l'ensemble du projet.

\section{Mise en relation des ressources et des résultats quantitatifs du projet}

. En observant les résultats de l'analyse, on peut faire l'hypothèse d'un effet d'apprentissage au niveau des coûts de développement de chaque cours. Une différence nette et progressive se marque en effet entre le prix de revient des premiers cours développés au sein du projet (environ $300000 €$ ) et les suivants (entre 50000 et $100000 €$ ), le coût moyen de développement d'un cours avoisinant les $120.000 €$.

Trois données peuvent être calculées : le coût moyen de la production d'une heure e-learning (coûts totaux divisés par le nombre d'heures développées), qui est de $2887 €$; le coût global du projet par apprenant : coûts totaux divisés par le nombre d'apprenants (étudiants et stagiaires), soit $447 €$; le coût d'une heure d'e-learning par apprenant : coût total divisé par (nombre d'apprenants $\mathrm{X}$ nombre d'heures suivies par apprenant), soit $14 €$. Ces valeurs n'ont guère de signification dans l'absolu : il faut les comparer dans le temps pour un même projet (ex. évolution du coût de production d'une heure e-learning au cours des années successives) ou entre différents projets. Ces calculs montrent que l'e-learning n'est intéressant que pour de grandes populations d'apprenants et de nombreuses heures de formation, et ce vu l'importance des ressources mobilisées pour développer des cours à distance.

\section{Analyse des résultats en termes d'apprentissages et de satisfaction}

La méthode d'analyse des résultats, en termes d'apprentissages et de satisfaction des participants, a été appliquée à un seul cours (management des personnes), lorsqu'il a été dispensé en entreprise. L'efficacité de la formation a été mesurée de façon quantitative au moyen de la comparaison des résultats des étudiants à deux tests similaires (pré-test et post-test), portant sur des compétences préalablement identifiées (e.g., questions concernant la capacité à encadrer des individus, la capacité à gérer des conflits). 
Les résultats des pré- et post-tests n'ont pas été significativement différents. Après questionnement des stagiaires, il apparaît que leurs compétences acquises ont été faibles dans les premiers groupes de test du cours, en raison d'une articulation insuffisante entre la théorie (documents à lire préalablement sur la plate-forme) et les exercices (jeux de rôles réalisés durant la partie présentielle). Cette faiblesse du cours en e-learning a fait l'objet d'une amélioration lors des sessions suivantes.

L'efficacité de la formation a également été vérifiée au moyen d'entretiens de groupes pour les participants, et d'entretiens individuels pour leur superviseur (le jour même, à chaud, et dix mois plus tard, à froid). Les entretiens avec les participants portaient sur :

- ce qu'ils avaient appris du cours ou ce dont ils se souvenaient ;

- ce qu'ils allaient appliquer ou avaient appliqué, une fois de retour au travail ;

- les ressources acquises durant le séminaire qui leur seraient utiles pour mettre en application les concepts ;

- les difficultés auxquelles ils ont eu à faire face.

L'interview menée auprès des superviseurs de l'entreprise permet de relever des indices de la mise en œuvre de nouvelles pratiques de gestion des personnes par les participants. Ces entretiens ont porté sur les impacts positifs qu'avait amené le séminaire au niveau de la compétence et de la performance des participants de retour à leur poste de travail (immédiatement après ou dix mois plus tard). Les réponses fournies témoignent d'une certaine efficacité de la formation car les compétences en progrès (tant dans l'évaluation à chaud que dans l'évaluation à froid) citées par les participants et par les superviseurs correspondent à celles fixées dans le programme du cours. Autrement dit, il se confirme, tant au niveau des participants que de leur superviseur, que les compétences d'encadrement des individus, de gestion des conflits, etc., se sont améliorées «sur le terrain» directement après la formation et dix mois plus tard. Ceci montre qu'il y a eu un transfert de compétences au poste de travail au sens de Kirkpatrick(1994).

\section{Conclusion : intérêts et limites de la démarche d'évaluation}

Si nous analysons la démarche d'évaluation du projet e-learning, construite dans un premier temps comme expérience-pilote, nous pouvons tirer les conclusions suivantes. Les intérêts de cette démarche sont nombreux :

- l'approche est non seulement focalisée sur les ressources mais aussi sur les résultats du projet ;

- elle permet de porter un regard différent (car plus global) et en recul (analyse des coûts et des résultats), sur le projet, ce qui apparaît bien nécessaire après cinq années de travail ; 
- elle permet une gestion professionnelle et systématique du projet ;

- elle donne un fil conducteur pour l'établissement de budgets, le développement futur du projet et la création de tableaux de bord synthétiques.

Par contre, certaines difficultés sont à prendre en considération :

- la méthode demande un temps considérable et de nombreuses ressources ;

- le manque d'information sur les coûts et les résultats, rend la démarche d'évaluation difficile et quelquefois approximative (certaines données, non disponibles, doivent être estimées).

La première difficulté ne s'effacera pas dans le futur, car cette démarche consomme du temps et des ressources. La seconde est davantage liée au caractère exploratoire et pilote de la démarche d'évaluation: en effet, si des indicateurs de résultats et des méthodes de recueil de ceux-ci sont prévus en début de projet, la collecte des informations devrait être facilitée.

En conclusion, nous pouvons dire qu'une démarche systématique d'évaluation d'un projet e-learning tant dans sa dimension économique que pédagogique, est très intéressante pour les gestionnaires des projets ou les pouvoirs publics qui les financent. En effet, ceux-ci peuvent disposer d'indicateurs, de points de repère, qui guident leur évaluation ex post (après la réalisation d'un projet) et leur prise de décision ex ante (opportunité de lancer un nouveau projet).

\section{Bibliographie}

Hulsman, T., «Costs without camouflage, a cost-analysis of Oldenburg university's two graduate certificate programs offered as part of the online master of distance", in U. Bernath, E. Rubin, Reflections on teaching and learning in an online master program, Oldenburg, University Carl von Ossietzky, Center for Distance Education, 2003, pp. 167226.

Kirkpatrick, D.L., Evaluating Training Programs: The Four Levels, San Fransisco, BerrettKoehler, 1994.

Ljosa, E., Credibility, legitimation and quality assurance in open distance learning, in $\mathrm{H}$. Hansson, C. Holmberg, Eight contributions on quality and flexible learning, Hämösand, Swedish agency for flexible learning, rapport $n^{\circ} 3,2003$, pp. 13-26.

Rekkedal, T. «Quality assurance in norwegian education. The background for NADE's quality standards with reference to some European initiatives», in H. Hansson, C. Holmberg, Eight Contributions on Quality and Flexible Learning, Hämösand, Swedish agency for flexible learning, rapport $n^{\circ} 3 / 2003$, 2003, pp. 27-54.

www.qual-elearning.net, E-learning quelles attentes en matière de qualité ? (consulté le $29 / 5 / 2008$ ) 
8 Distances et Savoirs. Volume X

www.be-odl.org, Consortium SEVAQ, outil d'évaluation de dispositifs de formation en ligne, projet européen Leonardo da Vinci. (consulté le 30/5/2008) 